\title{
Bioaffinity mass spectrometry screening using droplet-based microfluidics
}

\author{
Hoan Vu ${ }^{\mathrm{a}}$, Ronald J. Quinn ${ }^{\mathrm{a}^{*}}$, Nam-Trung Nguyen ${ }^{\mathrm{b} *}$ \\ * Correspondence to: \\ Nam-Trung Nguyen, Queensland Micro- and Nanotechnology Centre, Griffith University, Brisbane, \\ Qld 4111, Australia. Email: nam-trung.nguyen@griffith.edu.au \\ Ronald J Quinn, Eskitis Institute for drug discovery, Griffith University, Brisbane, Qld 4111, Australia. \\ Email: r.quinn@griffith.edu.au \\ ${ }^{a}$ Eskitis Institute, Griffith University, Brisbane, Qld 4111, Australia \\ ${ }^{\mathrm{b}}$ Queensland Micro- and Nanotechnology Centre, Griffith University, Brisbane, Qld 4111, Australia
}

\begin{abstract}
Bioaffinity mass spectrometry screening is a novel approach using non-denaturing electrospray ionisation mass spectrometry (ESI-MS) in identifying drug leads. The screening can detect and preserve noncovalent protein-active drug ligand complexes under different physiological conditions. Although there are many successful screening campaigns employing the technique, the big challenge of the screening is the reduction of sample volume needed. We demonstrate in this manuscript that analysis of samples can be performed using a microfluidic T-junction. Droplets of screening samples are produced and delivered directly into the electrospray emitter of the Fourier Transform mass spectrometer. The results show that a MS instrument with a conventional ESI source can clearly detect the samples and distinguish it with the separating oil phase. The proposed technique opens the possibility of bioaffinity mass spectrometry screening of samples with a simple microfluidic device.
\end{abstract}

Keywords: droplet-based microfluidics, compartmentalisation, ESI, bioaffinity mass spectrometry

\section{Introduction}

Bioaffinity mass spectrometry screening is a simple mix-and-measure assay using mass spectrometry. This method identifies active compound ligands binding with therapeutical target proteins under different physiological condition. We have previously applied the above screening method to successfully screen crude natural product extract and fragment compound libraries ${ }^{1,2}$. Although this is a very efficient and powerful tool for preliminary screenings, the consumption of limited target proteins and tested compounds still remains a big challenge. State-of-the-art automated systems such as Triversa Nanomate, allows the direct transfer of samples to an array of electrospray ionisation (ESI) nozzles. However, the relatively large nozzle size increases the cost of consumables making it prohibitive for a large spectrum of researchers. Recent advances in microfluidics and lab-on-a-chip (LOC) technologies allow the implementation of conventional lab protocols into a microchip. Efforts have also been made to use microfluidic technology for mass spectrometry based proteomics. ${ }^{[4]}$ Another common method is the use of droplet-based microfluidics. In droplet-based microfluidics, samples are compartmented in droplets that are protected by an immiscible oil phase. ${ }^{[5]}$ The droplets represent the reactors or the wells of the conventional microwell plates which reduce the volume consumption of samples.

In order to adapt droplet-based microfluidic technology for mass spectrometry screening, the major challenge is the interface of the microfluidic device to the conventional ESI-MS system. Droplets can first be formed in a microfluidic chip using two basic configurations such as the T-junction or the flowfocusing junction. ${ }^{[6]}$ The droplets then can be delivered to the mass spectrometry for screening. Recently, Trivedi proposed the use of of-the-shelf polytetrafluoroethylene (PTFE) tubing for the formation and handling of sample droplets. ${ }^{[7]}$ With PTFE tubing as storage and transport platform for droplets, a number of groups have proposed automated robotic platforms that convert samples from a micro well array into a droplet train. Clausell-Tormos et al. used an autosampler to load samples from a microtitre plate into a plug train in a PTFE tubing. ${ }^{[8]}$ The samples are encapsulated and separated by 2.5\% (v/v) perfluoro-octanol in fluorinated FC40 oil. This system was used for kinetic analyses of compound libraries based on optical detection. Wu et al. applied the same concept of automated sampling to transfer a droplet train from a well plate to a PTFE tubing and the to a PDMS microfluidic chip for subsequent manipulation. ${ }^{[9]}$ Gielen et al. reported a robotic sampling system based on a rotating oil filled carousel. ${ }^{[10]}$ Samples are transferred to a PTFE tubing from the bottom of sample 
tubes to minimise contamination. $0.5 \%(\mathrm{v} / \mathrm{v})$ perfluro-octanol in FC40 was also used as the immiscible oil phase.

The sample droplets of the above mentioned works were analysed using simple optical absorbance detection or capillary electrophoretic separation. ${ }^{[11]}$ A few attempts to interface droplet-based microfluidics with conventional mass spectrometry have been reported in the literature. Pereira et al. transferred the droplet train from the PTFE tubing to a matrix-assisted laser desorption/ionisation (MALDI) mass spectrometry ${ }^{[12]}$ The droplets with the MALDI matrix were deposited on the MALDI matrix, where the oil was removed by an oleophilic film. Fidalgo et al. were the first to couple dropletbased microfluidics to an ESI-MS system ${ }^{[13]}$ The droplets were transferred to an continuous aqueous stream before entering the emitter nozzle. Since the final stage of this concept is still based on continuous-flow microfluidics, the sample is diluted by Taylor dispersion. Zhu and Fang reported a similar concept of transferring sample droplet to a continuous flow and then to the emitter. ${ }^{[14]} \mathrm{Li}$ et al. were the first to introduce a droplet train in oil directly to a ESI-MS system. ${ }^{[15]}$ Hexane, FC-72, FC-77, FC-40 and perfluorodecalin (PFD) were tested as the immiscible phases. FC-40 and PFD showed minimum noise level and prove themselves as suitable for ESI experiments. The same group later use the robotic sampling approach to transfer samples from a well plate to the PTFE tubing to a the electrospray emitter. ${ }^{.16]}$ Recently, Smith et al. use a microfluidic device to deliver surfactant stabilised picoliter droplets to a ESI-MS system. ${ }^{[17]}$ Four different protein samples compartmented in droplets and separated by FC-3283 containing 2\% w/w PicoSurf-2 surfactant (Sphere Fluidics Ltd.) could be detected without cross contamination.

In this manuscript, we demonstrate the formation of sample droplet and direct coupling of a droplet train to an ESI-MS system for identifying protein-ligand complexes. We successfully detected the protein and the protein-ligand complex droplets using the microfluidic T-junction device. The manuscript demonstrates that a simple and low-cost interface setup with common fluorinated oil is suitable for providing mass spectrometry results of proteins and protein-ligand noncovalent complexes. Successful detection of the complexes is an important step for designing and coupling new multiple channel devices with ESI-MS for the development of a high-throughput bioaffinity mass spectrometry screening.

\section{Experimental}

Chemical and Reagents. The fluorinated oil FC-40 and PFOH $(1 \mathrm{H}, 1 \mathrm{H}, 2 \mathrm{H}, 2 \mathrm{H}$-perfluorooctan-1-ol), bovine carbonic anhydrase II (bCAII, EC 4.2.1.1, 29,089 Da), and sulfanilamide $\left(\mathrm{C}_{6} \mathrm{H}_{8} \mathrm{~N}_{2} \mathrm{O}_{2} \mathrm{~S}, 172 \mathrm{Da}\right.$ ) were purchased from Sigma Aldrich.

Sample Preparation. bCAII used without further purification. The protein was dissolved in ammonium acetate $(10 \mathrm{mM}, \mathrm{pH} 7)$ to generate the stock solution. The specific inhibitor of bCAII used in this study was sulfanilamide. It was dissolved in methanol to generate stock solutions. The protein was mixed with the inhibitor and incubated one hour at room temperature. The final inhibitor: protein ratio was $\sim 8: 1$.

Microfluidic device The microfluidic device is fabricated in Polymethylmethacrylate (PMMA) using standard laser ablation and thermal sealing technique. The width of the dispersed phase and continuous phase channel are $450 \mu \mathrm{m}$ and $800 \mu \mathrm{m}$ respectively. The microfluidic channels has a depth of about $1500 \mu \mathrm{m}$. A PTFE tubing (Upchurch scientific, Australia) is then inserted directly into the PMMA device through the end access hole and sealed using Ultraviolet (UV) glue to prevent any leakages.

\section{Formation of sample droplets using capillary tubings}

Initial experiments were carried out on the formation of a droplet train in fluorinated oil using a capillary T-junction device. The fluorinated oil is a mixture of FC40 and 30\% v/v PFOH. The aim is to stabilize the droplets and to prevent unspecific adsorption of biomolecules to surfaces of the fluidic system. PTFE tubings with an inner diameter and outer diameter of $1.56 \mathrm{~mm}$ and $0.15 \mathrm{~mm}$, respectively were purchased from Cole-Pamer (USA) and was used as the channels in our experiment. The two inlet tubings for the T-junction are $100 \mathrm{~mm}$ long. The outlet tubing for storage of the droplet train and interfacing to the ESI source is $400 \mathrm{~mm}$ long. The droplets are formed in a zero-dead volume stainless-steel internal T-junction fitting (ZT.5, Valco, Switzerland). The aqueous phase (protein sample) is delivered from a syringe (volume of $250 \mu \mathrm{L}$, inner diameter of $2.30 \mathrm{~mm}$, Hamilton). Another syringe (volume of $500 \mu \mathrm{L}$, inner diameter of $3.26 \mathrm{~mm}$, SGE) provides the oil phase to the 
tubing. Both syringes are run on the same syringe pump (World Precision Instruments, model sp200i-z, USA). Thus, the flow rate ratio in our droplet formation experiment were kept constant at $\left(\mathrm{Q}_{\text {oil }} / \mathrm{Q}_{\text {sample }}=2\right)$. The droplet formation process was observed and recorded by a high-speed camera (FASTCAM SA3, Photron, USA) mounted on an inverted microscope setup (ECLIPSE TS100-F, Nikon, Japan). Figure 1 shows the setup of the capillary T-junction device for the formation of the droplet train.

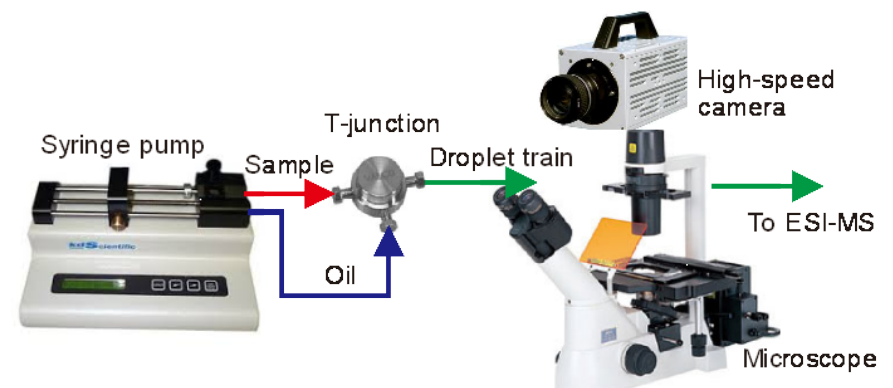

Figure 1 Experimental setup for the formation of sample droplets in a T-junction device with Teflon tubings as microfluidics channels

For interfacing with ESI-MS, droplet trains of protein samples were formed at a flow rate of $5 \mu \mathrm{L} / \mathrm{min}$. A sample droplet of $3 \mathrm{~nL}$ volume, separated by an oil plug of $6 \mathrm{~nL}$ volume are expected to be delivered through the PTFE tubing (i.d of $150 \mu \mathrm{m}$. After forming the droplet train, the outlet tubing is connected to the Apollo electrospray ESI source of a 12 Tesla Bruker Solarix Fourier Transformation Mass Spectrometer (ESI-FTMS). The droplet train was injected directly by a WPI syringe pump with a flow rate of $10 \mu \mathrm{L} / \mathrm{min}$. Ions were formed via electrospray ionization in the Apollo source employing an offaxis, grounded electrospray probe positioned about $1.5 \mathrm{~cm}$ from the metallized terminus of the glass desolvation capillary. Mass spectra were recorded in the positive ion mode with a mass range from 50 to $6000 \mathrm{~m} / \mathrm{z}$ for broadband low-resolution acquisition. Each spectrum was a scan composed of 256,000 data points. All aspects of pulse sequence control and data acquisition were performed on an Intel ${ }^{\circledR}$ Xeon® CPU W3520@2.67 GHz Quad Core computer running Bruker Solarix control software under Windows operating system. The end plate voltage was biased at $500 \mathrm{~V}$ and the capillary voltage at $4000 \mathrm{~V}$ relative to the ESI needle during data acquisition. Nebulizing nitrogen gas with a pressure of 1.5 bar and a counter-current drying nitrogen gas with a flow rate of $3 \mathrm{~L} / \mathrm{min}$ were employed. The drying gas temperature was maintained at $180^{\circ} \mathrm{C}$.

\section{Formation of sample droplets using PMMA T-junction microfluidic device}

Similarly experiments were carried out on the formation of a droplet train in a PMMA T-Junction microfluidic device. The device was made of Polymethyl methacrylate (PMMA) is durable against the organic surfactant perfluorooctanol. The droplets train was formed, observed in the device, and transported to the ESI source using PTFE tubing connection with an inner diameter and outer diameter of $0.5 \mathrm{~mm}$ and $1.58 \mathrm{~mm}$. The protein sample is delivered from a syringe (volume of $2.50 \mathrm{~mL}$, inner diameter of $7.28 \mathrm{~mm}$, Hamilton) and acts as the dispersed phase fluid. Another syringe (volume of $5.00 \mathrm{~mL}$, inner diameter of $10.30 \mathrm{~mm}$, Hamilton) provides the oil phase and acts as the continuous phase fluid. Both syringes are run on the same syringe pump (World Precision Instruments, model sp200i-z, USA). The flow rate ratio $\left(\mathrm{Q}_{\text {sample }} / \mathrm{Q}_{\text {oil }}\right)$ in our droplet formation experiments was performed at different values, $0.2,0.25,0.33$ and 0.5 .

\section{Results and Discussions \\ Optimisation of MS instrumental parameters}

Nondenaturing ESI-MS can preserve noncovalent protein-ligand complexes from solution phase into the gas phase. However the noncovalent complex is extremely fragile in the gas phase state. Excess heat or high collision energy in the atmospheric pressure or vacuum interface can dissociate complexes. Instrumental parameters can be optimized to stabilize the noncovalent complexes. The first important parameter of the ESI source is the capillary temperature. At a capillary temperature of $100^{\circ} \mathrm{C}$ non-covalent complexes are poorly desolvated and are thus poorly observed. Increasing the capillary temperature to $200^{\circ} \mathrm{C}$ will improve the desolvation process but will destabilize the complexes. To get the best signal intensity of the noncovalent complexes, this work was carried out with the capillary temperature at $180 \circ \mathrm{C}$. 
The second important parameter of the ESI source is the skimmer voltage. A higher skimmer voltage generally accelerates ions from the source region to the high vacuum analyser of the instrument, but it increases the internal energy imparted to the complex and can destabilize the complex. Experiments on different skimmer voltage values were conducted to find the optimum value for the best protein-ligand complex signal. Figure 2 shows the signal intensity of the protein-ligand complex decreases when the skimmer voltages increase from 50 to $60,70,80 \mathrm{~V}$. At skimmer voltage of $50 \mathrm{~V}, 60 \mathrm{~V}$ and $70 \mathrm{~V}$, the intensity of the [bCA-SFM] complex at charge state $11+$ is about $2.5 \times 10^{9}, 2 \times 10^{9}$, and $1 \mathrm{x} 10^{9}$, respectively. At skimmer voltage of $80 \mathrm{~V}$, the intensity of the complex was almost at null. Figure 3 shows the signal intensity of the protein-ligand complex at skimmer voltage of 50, 30 and $10 \mathrm{~V}$. The protein complex signal had the best signal sensitivity at skimmer voltage of $50 \mathrm{~V}$. When skimmer voltages were decrease to $30 \mathrm{~V}$ or $10 \mathrm{~V}$, nonspecific binding are apparent. Figure 4 illustrates the effect of the skimmer voltages to the protein-ligand complex. In order to obtain the best protein-ligand noncovalent complex, a skimmer voltage of $50 \mathrm{~V}$ was chosen as the optimal skimmer voltage for all the experiments in this manuscript.

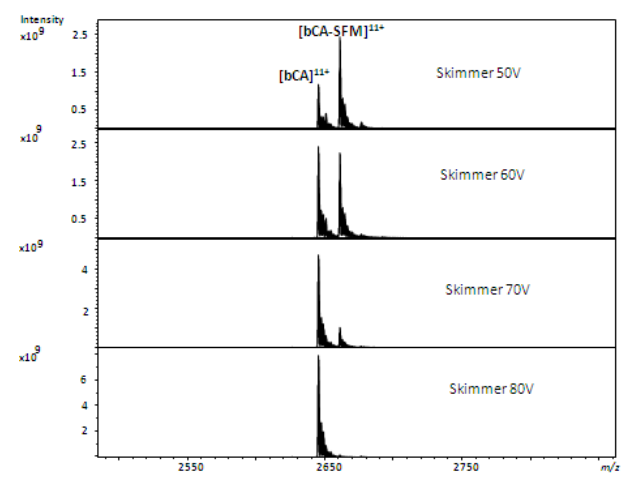

Figure 2 Mass spectra of the mixture of bCA II with sulfanilamide under skimmer voltages 50, 60, 70, and $80 \mathrm{~V}$. [bCA II-SFM] complex is dissociated under high skimmer voltage

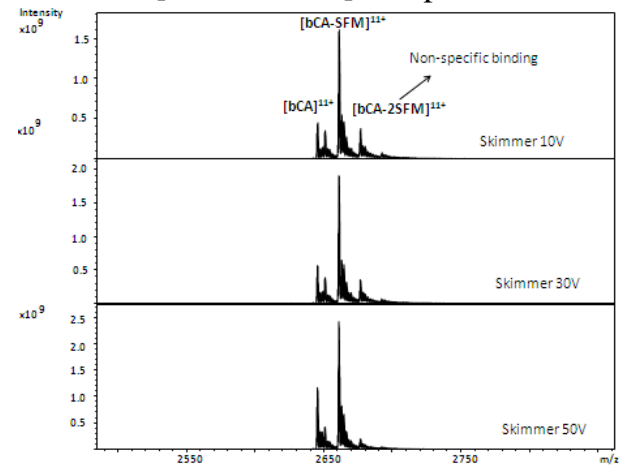

Figure 3 Mass spectra of the mixture of bCAII with sulfanilamide under skimmer voltages of 50, 30, and 10V.[bCAII-SFM] complex was better with skimmer voltage of 50V.

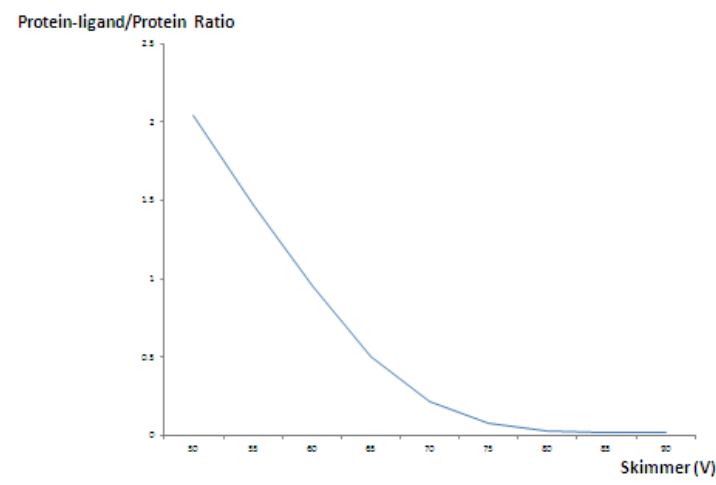

Figure 4 Effect of skimmer voltage to the protein-ligand complex. 
ESI-MS analysis of the sample train formed in the PTFE tubing of the T-junction device

The droplet train was formed after injecting both the protein sample $(3.4 \mu \mathrm{M})$ and the fluorinated oil into the T-junction device. Figure (5a) shows the total ion chromatography (TIC) of the droplet train Figure (5b) shows the mass spectrum of the FC40 oil. Figure (5c) shows the spectrum of the protein in three different charge states, $\mathrm{m} / \mathrm{z} 2424.9$ at $12+, \mathrm{m} / \mathrm{z} 2645.5$ at 11+, and $\mathrm{m} / \mathrm{z} 2909.9$ at $10+$. Figure 5d) shows the spectrum of the protein complex bCA-sulfanilamide at three different charge states, $\mathrm{m} / \mathrm{z}$ 2439.8 at $12+, \mathrm{m} / \mathrm{z} 2661.4$ at $11+$, and $\mathrm{m} / \mathrm{z} 2927.3$ at $10^{+}$. From the complex peak, the molecular weight of the binding inhibitor was identified:

$\mathrm{MW}_{\text {inhibitor }}=(\mathrm{m} / \mathrm{z}$ complex- $\mathrm{m} / \mathrm{z}$ protein $) \times \mathrm{z}=(2661.4-2645.7) \times 11=15.7 \times 11=172$
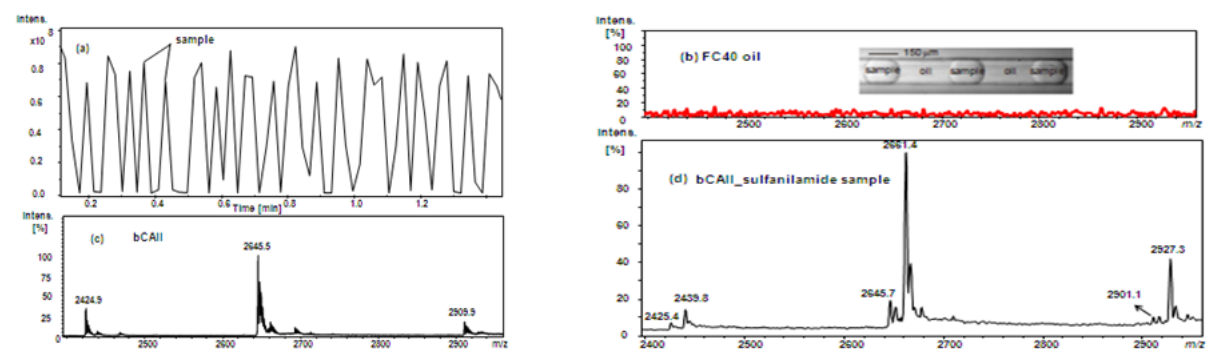

Figure 5 Preliminary results of interfacing a capillary T-junction with the ESI-MS system: (a) Total ion chromatography of the droplet train in an oil carrier. Mass spectra of (b) FC40 oil; (c) bCAII protein sample; (d) bCAII-sulfanilamide noncovalent complexes.

ESI-MS analysis of the sample droplets formed in the PMMA microfluidic T-junction device The protein sample and the oil were injected into the PMMA device using the same syringe pump. By keeping the dispersed phase flow rate constant at $500 \mu \mathrm{L} /$ hour, mass spectra were acquired at different flow rate ratio $\left(\mathrm{Q}_{\mathrm{D}} / \mathrm{Q}_{\mathrm{C}}\right)$ values. These ratios were $1 / 1,1 / 2,1 / 3,1 / 4$ and $1 / 5$. With the ratio $1 / 1$, the droplets were merged which is indicated by the peaks observed in the TIC. With the ratio $1 / 5$, the droplets high intensity was small as the droplets are significantly smaller. Experiment results (Figure 6) shows that the best flow rate ratio was $1 / 4$ with the dispersed phase flowrate at $500 \mu \mathrm{L} /$ hour and the continuous phase oil flow rate at $2 \mathrm{~mL} /$ hour. At these flow rates, the TIC of the samples are distinctive and 8 droplets are detected during the scan.

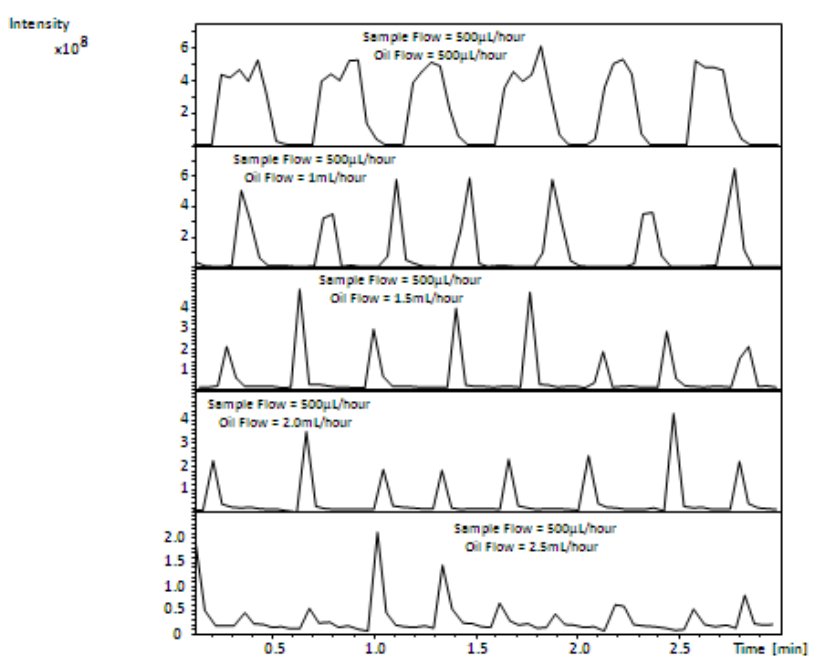

Figure 6 TIC of samples acquired with different flow rate ratio values by keeping the sample flow rate constant at $500 \mu \mathrm{L} /$ hour 
Next, by keeping the optimum flow rate ratio constant at $1 / 4$, experiments were performed at different values of sample flow rate and oil flow rate. Figure 7 shows the TIC of protein samples passing through five different settings. Setting 1 is sample flow rate at $250 \mu \mathrm{L} /$ hour and oil flow rate at $1 \mathrm{~mL} /$ hour. Setting 2 is sample flow rate at $375 \mu \mathrm{L} /$ hour and oil flow rate at $1.5 \mathrm{~mL} /$ hour. Setting 3 is sample flow rate at $500 \mu \mathrm{L} /$ hour and oil flow rate at $2 \mathrm{~mL} /$ hour. Setting 4 is sample flow rate at $625 \mu \mathrm{L} /$ hour and oil flow rate at $2.5 \mathrm{~mL} /$ hour. Setting 5 is sample flow rate at $725 \mu \mathrm{L} /$ hour and oil flow rate at $3 \mathrm{~mL} /$ hour. In setting 1 and 2, the droplets were flowing slowly and only limited samples are detected in a 3 minutes scan. In both setting 4 and 5, the droplets were flowing significantly faster than the scanning rate of the MS. Hence, distinctive droplets cannot be detected clearly. These results also set the limit of the proposed system. Setting 3 was chosen for experiments with the protein-ligand complex (bCA-SFM) as it detects the most number of sample droplets clearly. The complex bCA-SFM sample and the oil were injected into a similar PMMA T-junction device. The flow rate ratio is $1 / 4$ with the sample flow rate $500 \mu \mathrm{L} /$ hour and the oil $2 \mathrm{~mL} /$ hour. Figure 8 shows the TIC of the protein complex sample and its spectrum.

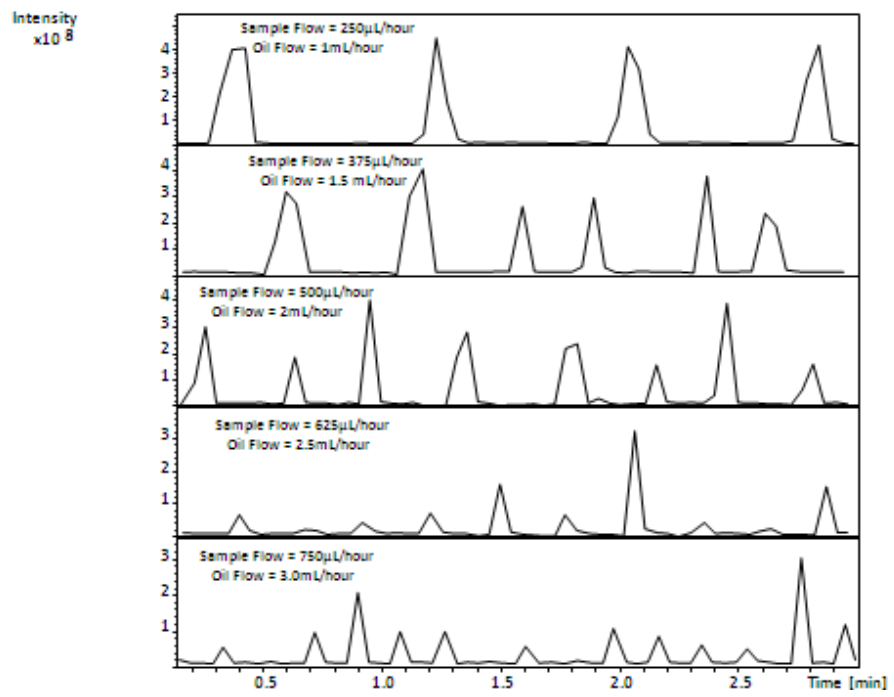

Figure 7 TIC of samples acquired with constant flow rate ratio $\left(Q_{\text {sample }} / Q_{\text {oil }}=1 / 4\right)$ while changing both the sample and the oil flow rates

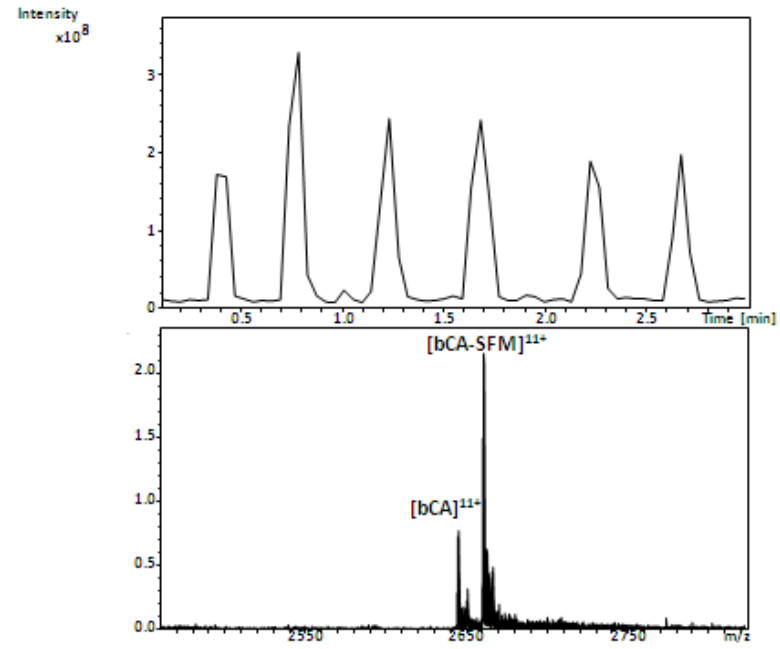

Figure 8 TIC of (bCA-SFM) protein complex acquired at flow rate ratio $\left(\mathrm{Q}_{\text {sample }} / \mathrm{Q}_{\mathrm{oil}}=1 / 4\right)$ with sample flow rate $500500 \mu \mathrm{L} /$ hour and the oil $2 \mathrm{~mL} /$ hour 


\section{Conclusions}

In this manuscript, we have demonstrated a low-cost and simple method for compartmenting protein samples in fluorinated oil for subsequent analysis with a conventional ESI-MS system. The results show a detection of a protein-ligand complex can take place in a very short time (seconds) and with a small sample consumption. For the PMMA T-junction Junction microfluidic device, a flow rate of 500 $\mu \mathrm{L} /$ hour and $2 \mathrm{~mL} /$ hour for the protein sample and the separated oil, respectively are the best values for the droplet train. A skimmer voltage of $50 \mathrm{~V}$ is the optimal parameter of the ESI source for our microfluidic-MS system. The method shows the proof of concept for droplet-based drug screening using mass spectrometry. The sample droplets could be transferred to the tubing directly from a microwell plate using a robotic platform or a specially designed parallel to serial sampling microfluidic device. With the later concept, droplet merging and reaction could be integrated on the same chip for the ease of handling and the required high throughput.

\section{Acknowledgment}

The research leading to these results was supported by the start-up grant of Griffith University and a equipment grant of the Griffith University Research Infrastructure Program awarded to NTN. HV and RQ were supported by .....

\section{References}

[1] H. Vu and R.J. Quinn, Direct screening of natural product extracts using mass spectrometry, $J$. Biomol. Screening 2008, 13, 265-275

[2] H.Vu, C. Rouiller, M. Campitelli, K. R. Trenholme, D. L. Gardiner, K. T. Andrews, T. SkinnerAdams Tina, G. J. Crowther, W. C. Van Voorhis, R. J. Quinn, Plasmodium Gametocyte Inhibition Identified from a Natural-Product-Based Fragment Library, ACS Chem. Biol.,2013, 8 2654-2659

[3] http://www.advion.com/products/triversa-nanomate/ (accessed on 24.09.2013)

[4] J. Lee, S. A. Soper, K. K. Murray, Microfluidic chips for mass spectrometry-based proteomics, J. Mass. Spectrom. 2009, 44, 579-593.

[5] A. B. Theberge, F. Courtois, Y. Schaerli, M. Fischlechner, C. Abell, F. Hollfelder, W. T. S. Huck, Microdroplets in Microfluidics: An Evolving Platform for Discoveries in Chemistry and Biology, Angw. Chem., Int. Ed. 2010, 49, 203-214.

[6] S. Teh, R. Lin, L. Hung, A. P. Lee, Droplet microfluidics, Lab Chip 2008, 8, 198-220.

[7] V. Trivedi, A. Doshi, G. K. Kurup, E. Ereifej, P. J. Vandevord, A . S. Basu, A modular approach for the generation, storage, mixing, and detection of droplet libraries for high throughput screening, Lab Chip 2010, 10, 2433-2442

[8] J. Clausell- Tormos, A. D. Griffiths, C. A. Merten, An automated two-phase microfluidic system for kinetic analyses and the screening of compound libraries, Lab Chip 2010, 10, 1302-1307 [9] J. Wu, M. Zhang, Z. Li, W. Wen, Multiple and High-Throughput Droplet Reactions via Combination of Microsampling Technique and Microfluidic Chip, Anal. Chem. 2012, 84, 9689-9693

[10] F. Gielen, L. van Vliet, B. T. Koprowski, S. R. A. Devenish, M. Fischlechner, J. B. Edel, X. Niu, A. J. de Mello, F. Hollfelder, A Fully Unsupervised Compartment-on-Demand Platform for Precise Nanoliter Assays of Time-Dependent Steady-State Enzyme Kinetics and Inhibition, Anal. Chem. 2013, 85, 4761-769

[11] X. Niu, F. Pereira, J. B. Edel, A. J. de Mello, Droplet-Interfaced Microchip and Capillary Electrophoretic Separations, Anal. Chem. 2013, 85, 8654-8660

[12] F. Pereira, X. Niu, A. J. de Mello, A Nano LC-MALDI Mass Spectrometry Droplet Interface for the Analysis of Complex Protein Samples, PLOS One 2013, 8, e63087

[13] L. M. Fidalgo, G. Whyte, B. T. Ruotolo, J. L. P. Benesh, F. Stengel, Coupling Microdroplet Microreactors with Mass Spectrometry: Reading the Contents of Single Droplets Online, Angw. Chem., Int. Ed. 2009, 48, 3665-3668.

[14] Y. Zhu, Q. Fang, Integrated Droplet Analysis System with Electrospray Ionization-Mass Spectrometry Using a Hydrophilic Tongue-Based Droplet Extraction Interface, Anal. Chem. 2010, 82, 8361-8366.

[15] Q. Li, J. Pei, P. Song, R. T. Kennedy, Fraction Collection from Capillary Liquid Chromatography and Off-line Electrospray Ionization Mass Spectrometry Using Oil Segmented Flow, Anal. Chem. 2010, 82, 5260-5267. 
[16] S. Sun, T. R. Slaney, R. T. Kennedy, Label Free Screening of Enzyme Inhibitors at Femtomole Scale Using Segmented Flow Electrospray Ionization Mass Spectrometry, Anal. Chem. 2012, 84, 57945800 .

[17] C. A. Smith, X. Li, T. H. Mize, T. D. Sharpe, E. I. Graziani, C. Abell, W. T. S. Huck, Sensitive, High Throughput Detection of Proteins in Individual, Surfactant-Stabilized Picoliter Droplets Using Nanoelectrospray Ionization Mass Spectrometry Anal. Chem. 2013, 85, 3812-3816. 